\title{
A CONSEQUENCE OF ESSENTIAL SPECTRUM OF AREA ZERO OF ESSENTIALLY $\mathcal{A}_{1,1}$ OPERATORS
}

\author{
To the memory of my grandmother, Maria
}

\section{VASILE LAURIC}

Department of Mathematics

Florida A\&M University

Tallahassee, FL 32307

USA

e-mail: Vasile.Lauric@famu.edu

\begin{abstract}
We define the class that essentially $\mathcal{A}_{1,1}$ operators and prove that for such operators with zero area of the essential spectrum are essentially normal.
\end{abstract}

\section{Introduction}

Let $\mathcal{H}$ be a complex, separable, infinite dimensional Hilbert space, and let $\mathcal{L}(\mathcal{H})$ denote the algebra of all linear bounded operators on $\mathcal{H}$, and let $\mathbb{K}$ denote the two-sided ideal of all compact operators on $\mathcal{H}$. An operator $T \in \mathcal{L}(\mathcal{H})$ is called essentially normal if $D_{T}:=T^{*} T-T T^{*}$ is a compact operator, and $T$ is called $\alpha$-hyponormal (essentially -hyponormal) if $D_{T}^{\alpha}:=\left(T^{*} T\right)^{\alpha}-\left(T T^{*}\right)^{\alpha}$ is a positive definite operator 2010 Mathematics Subject Classification: Primary 47B20.

Keywords and phrases: essentially $\mathcal{A}_{1,1}$ operators, essential spectrum of area zero.

Received March 29, 2018; Revised April 30, 2018

(ㄷ) 2018 Scientific Advances Publishers 
( $D_{T}^{\alpha}$ is the sum of a positive definite operator and a compact operator, or equivalently, $\pi(T)$ is an $\alpha$-hyponormal operator in the Calkin algebra $\mathcal{L}(\mathcal{H}) / \mathbb{K}$, where $\pi: \mathcal{L}(\mathcal{H}) \rightarrow \mathcal{L}(\mathcal{H}) / \mathbb{K}$ is the canonical map), respectively. An operator $T \in \mathcal{L}(\mathcal{H})$ is of class $\mathcal{A}_{s, t}$ (notation $T \in \mathcal{A}_{s, t}(\mathcal{H})$ ) if $C_{s, t}^{T}:=\left|T_{s, t}\right|^{\frac{t}{s+t}}-|T|^{2 t}$ is a positive semidefinite operator and essentially $\mathcal{A}_{s, t}$ operator (notation $\left.T \in \mathcal{A}_{1,1}^{e}(\mathcal{H})\right)$ if $C_{s, t}^{T}$ is the sum of a positive semidefinite operator and a compact operator, where $T_{s, t}=|T|^{s} U|T|^{t}$, $s, t>0$, is the generalized Aluthge transform. Furthermore, for $T$ in $\mathcal{L}(\mathcal{H})$, let $\sigma(T)$ and $\sigma_{e}(T)$ denote the spectrum and the essential spectrum of $T,\|T\|_{e}$ the norm of $\pi(T)$ in the Calkin algebra, and $\sigma_{w}(T)$ the Weyl spectrum of $T$, that is the union of $\sigma_{e}(T)$ and the bounded components of $\mathbb{C} \sigma_{e}(T)$ that are associated with nonzero Fredholm index.

It is well-known that for a fixed $\alpha>0, \alpha$-hyponormal operators are class $\mathcal{A}_{s, t}$ operators for all $s, t>0$ and for $\alpha \leq 1$, Putnam's inequality holds, that is

$$
\left\|D_{T}^{\alpha}\right\| \leq \frac{1}{\pi} \mu_{2 \alpha}(\sigma(T)),
$$

where $\mu_{\beta}(\sigma)=\frac{\beta}{2} \iint_{\sigma} \rho^{\beta-1} d \rho d \theta$. In particular, for $\alpha=1$, one obtains the classical Putnam's inequality [5] that involves $\mu_{2}(\sigma(T))$, that is the area measure of the spectrum of $T$, and which holds the nice consequence that a hyponormal operator with spectrum or area zero must be a normal operator.

In [2], the following extension of Putnam's inequality was proved. 
Theorem A ([2], Theorem 3.1). If $T \in \mathcal{A}_{s, t}(\mathcal{H})$ and $s, t \leq 1$, then

$$
\begin{aligned}
\left\|\left|T_{s, t}\right|^{\frac{2 \min \{s, t\}}{s+t}}-|T|^{2 \min \{s, t\}}\right\| & \leq\left\|\left|T_{s, t}\right|^{\frac{2 \min \{s, t\}}{s+t}}-\left|T_{s, t}^{*}\right|^{\frac{2 \min \{s, t\}}{s+t}}\right\| \\
& \leq \frac{\min \{s, t\}}{\pi} \iint_{\sigma(T)} r^{2 \min \{s, t\}-1} d r d \theta .
\end{aligned}
$$

Since $\left|T_{1,1}\right|=\left|T^{2}\right|$, and if $T \in \mathcal{A}_{1,1}$, then

$$
\left\|\left|T^{2}\right|-|T|^{2}\right\| \leq\left\|\left|T_{1,1}\right|-\left|T_{1,1}^{*}\right|\right\| \leq \frac{1}{\pi} \mu_{2}(\sigma(T))
$$

According to ([3], Corollary 2.2), if $T \in \mathcal{A}_{s, t}(\mathcal{H})$ and $T_{s, t}$ is a normal operator, then $T$ is also a normal operator. Thus, a remarkable consequence of the Theorem A results.

Corollary B. If $T \in \mathcal{A}_{1,1}(\mathcal{H})$ and $\mu_{2}(\sigma(T))=0$, then the operator $T$ must be normal.

It is the purpose of this note to obtain a further consequence concerning essentially $\mathcal{A}_{1,1}$ operators.

Theorem. If $T \in \mathcal{A}_{1,1}^{e}(\mathcal{H})$ and $\mu_{2}\left(\sigma_{e}(T)\right)=0$, then the operator $T$ is essentially normal.

Proof. Let $T \in \mathcal{A}_{1,1}^{e}(\mathcal{H})$ and let $\mathcal{A}_{T}$ be the unital $C^{*}$-algebra generated by $\pi(T)$ in the Calkin algebra. Let $\rho: \mathcal{A}_{T} \rightarrow \mathcal{L}(\mathcal{K})$ be a faithful representation and denote $\rho(\pi(T))$ by $Q_{T}$. Since $\mathcal{A}_{T}$ is a unital algebra, $\sigma\left(Q_{T}\right)=\sigma_{e}(T)$ (according to [4], Proposition 1.3) and since $\rho$ is an isometric ${ }^{*}$-isomorphism, $C_{1,1}^{Q_{T}}=\rho\left(C_{1,1}^{\pi(T)}\right), Q_{T} \in \mathcal{A}_{1,1}(\mathcal{K})$. According to Corollary B, the operator $Q_{T}$ is normal and which concludes the proof. 
Since almost $\alpha$-hyponormal operators (that is operators for which $D_{T}^{\alpha}$ can be written as the sum of a positive semidefinite operator and a trace class operator) with $\alpha \leq 1$ and whose Weyl spectrum has area zero must be almost normal, that is its selfcommutator is trace class (see [1]), the following is a natural question to ask. An operator $T \in \mathcal{L}(\mathcal{H})$ is called almost $\mathcal{A}_{1,1}$ operator (notation $T \in \mathcal{A}_{1,1}^{\alpha l}(\mathcal{H})$ ) if $\left|T^{2}\right|-|T|^{2}$ is the sum of a positive semidefinite operator and a trace class operator.

Question. If $T \in \mathcal{A}_{1,1}^{a l}(\mathcal{H})$ and $\mu_{2}\left(\sigma_{w}(T)\right)=0$, then is $\left|T^{2}\right|-|T|^{2}$ a trace class operator? If so, is the trace zero?

\section{References}

[1] Vasile Lauric, Almost $\alpha$-hyponormal operators with Weyl spectrum of area zero, Int. J. Math. Math. Sci. 2011 (2011), pages 5; Article ID 801313, MR2794898.

DOI: http://dx.doi.org/10.1155/2011/801313

[2] S. M. Patel, M. Cho, K. Tanahashi and A. Uchiyama, Putnam's inequality for class $A$ operators and an operator transform by Cho and Yamazaki, Sci. Math. Jpn. 67(3) (2008), 393-401, MR2428060.

[3] S. M. Patel, K. Tanahashi, A. Uchiyama and M. Yanagida, Quasinormality and Fuglede-Putnam theorem for class $A(s, t)$ operators, Nihonkai Math. J. 17(1) (2006), 49-67, MR2241359.

[4] C. M. Pearcy, Some recent developments in operator theory, Amer. Math. Soc., Providence, R.I. 36 (1978), 73 pp.

$$
\text { DOI: http://dx.doi.org/10.1090/cbms/036 }
$$

[5] C. R. Putnam, An inequality for the area of hyponormal spectra, Math. Z. 116(4) (1970), 323-330, MR0270193.

DOI: https://doi.org/10.1007/BF01111839 